\title{
Differentiation of Fourier Series via Orthogonal Derivative
}

\author{
R. Cruz-Santiago, J. López-Bonilla and R. López-Vázquez \\ ESIME-Zacatenco, ICE Instituto Politécnico Nacional \\ Edif.5, 1er. Piso, Col. Lindavista, CP 07738, México DF \\ E-mail: jlopezb@ipn.mx
}

\begin{abstract}
It is very known that if the operator $\frac{d}{d x}$ acts on each term into a convergent Fourier Series (FS), then it may result a divergent series. This situation is remedied applying the symmetric derivative to FS, which implies the existence of the important Fejér-Lanczos Factors. In this paper, we show that the orthogonal derivative also leads to these Factors.
\end{abstract}

Keywords: Orthogonal derivative, least squares method, Fejér-Lanczos $\sigma$-factors, differentiation of FS

\section{INTRODUCTION}

If on the Fourier series (Lanczos 1966):

$f(x)=\frac{1}{2} a_{0}+\sum_{k=1}^{\infty}\left[a_{k} \cos (k x)+\mathrm{b}_{\mathrm{k}} \sin (k x)\right]$,

convergent in $[-\pi, \pi]$, we apply the operator $\frac{d}{d x}$ results:

$\frac{d}{d x} f(x)=\sum_{k=1}^{\infty} k\left[-a_{k} \sin (k x)+b_{\mathrm{k}} \cos (k x)\right]$

which it may be divergent (Gibbs 1898, Lanczos 1956). This problem was remedied by Lanczos (1956) with $f^{\prime}(x)$ defined as a Symmetric derivative (Aull 1967, Washburn 2006):

$f^{\prime}(x) \equiv \lim _{n \rightarrow \infty} \frac{1}{\frac{2 \pi}{n}}\left[f_{n}\left(x+\frac{\pi}{n}\right)-f_{n}\left(x-\frac{\pi}{n}\right)\right]$

with the partial sums:

$f_{n}(x)=g_{n}(x)+h_{n}(x)$,

$g_{n}(x)=\frac{1}{2} a_{0}+\sum_{k=1}^{n} a_{k} \cos (k x), h_{n}(x)=\sum_{k=1}^{n} b_{k} \sin (k x)$,

resulting the convergent expression:

$f^{\prime}(x)=\lim _{n \rightarrow \infty} \sum_{k=1}^{n} \sigma_{k} \frac{d}{d x}\left[a_{k} \cos (k x)+b_{\mathrm{k}} \sin (k x)\right]$,

with the Fejér-Lanczos Factors (Lanczos 1956, 1961, Jerri 2000):

$\sigma_{0}=1, \sigma_{k}=\frac{\sin \left(\frac{k \pi}{n}\right)}{\frac{k \pi}{n}}, k=1, \ldots, n, \sigma_{n}=0$.

The set of factors $\sigma_{k}$, for a given $n$, is equivalent to a discrete sampling function.

In (2) and (3) we employ two derivatives, however, also there is the orthogonal derivative (Washburn 2006, Groetsch 1998, Shen 1999, Hicks \& Liebrok 2000, Burch et al. 2005, López-

Bonilla et al. 2010, López-Bonilla et al. 2010, Diekema \& Koornwinder 2012) obtained by Lanczos (1956),
Cioranescu (1938) and Haslam-Jones (1953), hence it is natural to ask if this ultimate derivative leads to relation (5). The answer is yes, to see the next section.

\section{THE ORTHOGONAL DERIVATIVE}

Lanczos (Lanczos 1956, Gordon 2009) used the least squares method of Legendre (1806), Gauss (1809), Laplace (1812) to obtain an integral expression for the derivative of a function, that is, differentiation by integration:

$F^{\prime}(x)=\lim _{\epsilon \rightarrow 0} \frac{3}{2 \epsilon^{3}} \int_{-\epsilon}^{\epsilon} t F(x+t) d t$,

which may be applied to Fourier case:

$\frac{3}{2 \epsilon^{3}} \int_{-\epsilon}^{\epsilon} t g_{n}(x+t) d t \stackrel{(4)}{=} \frac{3}{2 \epsilon^{3}} \sum_{k=1}^{n} a_{k} \int_{-\epsilon}^{\epsilon} t \cos (k x+$

kt) $d t$,

$=-3 \sum_{k=1}^{n} a_{k} \frac{\sin (k x)}{k^{2}} A_{k} \quad$ with $\quad A_{k}(\epsilon)=\frac{1}{\epsilon^{3}}[\sin (k \epsilon)-$ $k \epsilon \cos (k \epsilon)]$;

Similarly:

$\frac{3}{2 \epsilon^{3}} \int_{-\epsilon}^{\epsilon} t h_{n}(x+t) d t \stackrel{(4)}{=} \frac{3}{2 \epsilon^{3}} \sum_{k=1}^{n} b_{k} \int_{-\epsilon}^{\epsilon} t \sin (k x+$

$k t) d t$,

$=3 \sum_{k=1}^{n} b_{k} \frac{\cos (k x)}{k^{2}} A_{k}$.

Therefore, the Lanczos derivative applied to partial sum (4) gives, taking $\epsilon=\frac{\pi}{n}$ :

$f^{\prime}(x)=\lim _{n \rightarrow \infty} \frac{3}{2 \epsilon^{3}} \int_{-\in}^{\epsilon} t f_{n}(x+t) d t$,

(8) and (9)
$=\lim _{n \rightarrow \infty} 3 \sum_{k=1}^{n} \frac{1}{k^{2}} A_{k}\left[-a_{k} \sin (k x)+\right.$

$\left.b_{k} \cos (k x)\right]$, 


$$
=\lim _{n \rightarrow \infty} \sum_{k=1}^{n} \frac{3 A_{k}}{k^{3}} \frac{d}{d x}\left[a_{k} \cos (k x)+b_{k} \sin (k x)\right],
$$

but the Bernoulli-Hôpital rule permits to observe the behavior:

$A_{k}\left(\epsilon=\frac{\pi}{n}\right) \underset{n>>1}{\longrightarrow} \frac{k^{3}}{3} \frac{\sin (k \epsilon)}{k \epsilon}=\frac{k^{3}}{3} \frac{\sin \left(\frac{k \pi}{n}\right)}{\frac{k \pi}{n}} \stackrel{\text { (6) }}{=}$

$\frac{k^{3}}{3} \sigma_{k}$

and this value employed in (10) implies (5), q.e.d. Thus, it is proved that the symmetric and Cioranescu-(HaslamJones)-Lanczos derivatives imply the same expression for the derivative of an infinite Fourier series, with the important participation of the Fejér-Lanczos factors.

\section{REFERENCES}

Aull, C. 1967. The first symmetric derivative. Am. Math. Monthly 74(6): 708-711.

Burch, N., Fishback, P.E., Gordon, R. 2005. The leastsquares property of the Lanczos derivative. Maths. Mag. 78(5): 368-378.

Cioranescu, N. 1938. La generalization de la premiére formule de la moyenne. Enseign. Math. 37: 292-302.

Diekema, E. and Koornwinder, T.H. 2012. Differentiation by integration using orthogonal polynomials, a survey. J. of Approximation Theory 164: 637-667.

Gauss, C.F. 1809. Theoria motus corporum coelestium in sectionibus conicis solem ambientium. Göttingen : 205-224.

Gibbs, J.W. 1898. Fourier's series. Nature 59: 200.

Gordon, R. 2009. A least squares approach to differentiation. Real Analysis Exchange 35(1): 205228.

Gottlieb, D., Chi-Wang, S. 1997. On the Gibbs phenomenon and its resolution. SIAM Rev. 39(4): 644-668.

Groetsch, C.W. 1998. Lanczos generalized derivative. Am. Math. Monthly 105(4): 320-326.

Haslam-Jones, U.S. 1953. On a generalized derivative. Quart. J. Math. Oxford Ser. 2(4): 190-197.

Hewitt, E., Hewitt, R.E. 1979. The Gibbs-Wilbraham phenomenon: An episode in Fourier analysis. Arch. Hist. Exact Sci. 21: 129-160.

Hicks, D.L., Liebrok, L.M. 2000. Lanczos generalized derivative: Insights and Applications. Applied Maths. and Compt. 112(1): 63-73.

Jerri, A.J. 2000. Lanczos-like $\sigma$-factors for reducing the Gibbs phenomenon in general orthogonal expansions and other representations. J. Comp. Anal. Appls 2: 1-17.

\section{CONCLUSION}

The orthogonal derivative is a generalization of the symmetric derivative and this is a generalization of the standard derivative. The orthogonal derivative acts as a smoothing (integrating filter): That is why the highest frequencies of the input are being suppressed in the deduction, and so this creates also the suppression of the Gibbs phenomenon (Lanczos 1956, 1961, 1966, Jerri 2000, Hewitt \& Hewitt 1979, Gottlieb \& Chi-Wang 1997, Jerri 2007). The Legendre polynomials (Lanczos 1956, 1966, Legendre 1785, Lanczos 1973) can be employed to extend the method of Cioranescu- (HaslamJones)-Lanczos to cover orthogonal derivatives of higher orders (Diekema \& Koornwinder 2012, Rangarajan \& Purushothaman 2005).

Jerri, A.J. 2007. The Gibbs phenomenon. Sampling Pub., New York, USA.

Lanczos, C. 1956. Applied analysis. Prentice-Hall, New Jersey, USA.

Lanczos, C. 1961. Linear differential operators. D. Van Nostrand Co., London, UK.

Lanczos, C. 1966. Discourse on Fourier series. Oliver \& Boyd, London, UK.

Lanczos, C. 1973. Legendre versus Chebyshev polynomials, in 'Topics in Numerical Analysis', (Proc. Roy. Irish Acad. Conf. on Numerical Analysis, Aug. 14-18, 1972), Ed. J. J. H. Miller, Academic Press, London, UK: 191-201.

Laplace, P.S. 1812. Théorie analytique des probabilities. Paris, Chap. 4.

Legendre, A.M. 1785. Recherches sur l'attraction des spheroids homogénes, Mémoires de Mathématiques et Physique. Acad. Roy. Sci. Paris 10: 411-435.

Legendre, A.M. 1806. Nouvelles methods pour la determination orbites des cometes, Paris.

López-Bonilla J., Rangel, A., Zuñiga-Segundo, A. 2010. Derivada generalizada de Lanczos en una discontinuidad finita. Ini. Inv. (Univ. of Jaén, Spain) 5(a4): 1-5.

López-Bonilla, J., Rivera, J., Vidal, S. 2010. Lanczos derivative via a quadrature method. Int. J. Pure Appl. Sci. Technol. 1(2): 100-103.

Rangarajan, S.K., Purushothaman, S.P. 2005. Lanczos generalized derivative for higher orders. J. Comp. Appl. Maths. 177(2): 461-465.

Shen, J. 1999. On the Lanczos generalized derivative. Am. Math. Monthly 106(8): 766-768.

Washburn, L. 2006. The Lanczos derivative, Senior Project Archive, Department of Mathematics, Whitman College, USA. 